\title{
A SEMIGROUP ANALOGUE OF FOGUEL'S COUNTEREXAMPLE
}

\author{
EDWARD W. PACKEL ${ }^{1}$
}

1. Introduction. In [1] Foguel provides a counterexample to Nagy's question of whether every power bounded operator on a Hilbert space is similar to a contraction. Generally questions about powers of operators have exponential analogues which can be phrased as questions about $\left(C_{0}\right)$ semigroups. The purpose of this note is to provide a counterexample to the semigroup analogue of Nagy's question - that is, to construct a uniformly bounded $\left(C_{0}\right)$ semigroup on a Hilbert Space $H$ whose generator is not similar to a dissipative operator. (An operator $L$ is dissipative on $H$ if $\langle L y, y\rangle$ $+\langle y, L y\rangle \leqq 0$ for all $y \in$ Domain $(L)$.) The method used depends strongly on Foguel's ideas and utilizes the viewpoint presented in Halmos' note [2] on Foguel's counterexample.

2. A necessary condition for similarity to a dissipative operator. Let $S(t)$ denote a $\left(C_{0}\right)$ semigroup and $L$ its infinitesimal generator on $H$. We will need the characterization of dissipative semigroup generators presented by Phillips [3, p. 203] which says that $L$ is dissipative if and only if $\|S(t)\| \leqq 1$ for all $t \geqq 0$.

Paralleling Foguel we define

$$
W(L)=\{x \in H \text { : weak limit }(t \rightarrow \infty) S(t) x=0\} .
$$

As a necessary condition for similarity to a dissipative operator we prove:

Lemma 1. If $L$ is similar to a dissipative operator, then $W(L) \cap\left[W\left(L^{*}\right)\right]^{\perp}=\{0\}$.

Proof. The argument is broken down into three parts in the manner of Halmos.

Part I. If $A$ generates a group of unitary operators $U(t),-\infty<t$ $<\infty$, then $W(A)=W\left(A^{*}\right)$ where $A^{*}$ generates the group $U^{*}(t)$. To see this, represent $U(t)$ as multiplication by $u^{t}$ on some $L_{2}(\mu)$ isometrically isomorphic to $H$, where $u$ is a measurable function of constant modulus one. Showing $W(A) \subseteq W\left(A^{*}\right)$ means showing that

Received by the editors March 18, 1968.

1 This work is a part of the author's doctoral dissertation submitted to the Massachusetts Institute of Technology in August 1967. The author wishes to thank Professor Gilbert Strang for his help and encouragement. 
$\int u^{t} f \bar{g} d \mu \rightarrow 0$ for all $g \in L_{2}(\mu)$ implies $\int \bar{u}^{t} f \bar{h} d \mu \rightarrow 0$ for all $h \in L_{2}(\mu)$. This follows by setting, for each $h, g=(\operatorname{sgn} f)^{2} \bar{h}$ and taking complex conjugates. By symmetry $W\left(A^{*}\right) \subseteq W(A)$, so equality holds.

Part II. If $D$ is dissipative and hence generates a contraction semigroup $C(t)$, then $W(D)=W\left(D^{*}\right)$. For this argument, the theory of strong unitary dilations $[4$, p. 15] yields a strongly continuous group $U(t)$ of unitary operators on a Hilbert space $H^{\prime} \supset H$ such that $\left.P U(t)\right|_{H}=C(t)$ for all $t \geqq 0$ where $P$ is the orthogonal projection of $H^{\prime}$ onto $H$. Furthermore $H^{\prime}$ is minimal in the sense that it is spanned by the elements $\{U(t) x\}, x \in H$ and $-\infty<t<\infty$. Define $H_{x}$ $=\left\{y \in H^{\prime}:\langle U(t) x, y\rangle \rightarrow 0\right.$ as $\left.t \rightarrow \infty\right\}$. For $x \in W(D),\langle C(t) x, y\rangle$ $=\langle P U(t) x, y\rangle=\langle U(t) x, P y\rangle=\langle U(t) x, y\rangle \rightarrow 0$ as $t \rightarrow \infty$ for all $y \in H$. Hence $H \subseteq H_{x}$ whenever $x \in W(D)$. Furthermore, $H_{x}$ is a linear manifold invariant under $U(t)$ and $U^{*}(t),-\infty<t<\infty$, and since $H^{\prime}$ is minimal as stated above, it follows that $x \in W(D)$ implies $H_{x}=H^{\prime}$. Call $A$ the generator of $U(t)$ and note that we then have $W(D)$ $\subseteq W(A) \cap H$ where $W(A)$ is defined on $H^{\prime}$. Conversely, $x \in W(A) \cap H$ implies $(U(t) x, y) \rightarrow 0$ as $t \rightarrow \infty$ for all $y \in H^{\prime}$. Thus for $y \in H,(C(t) x, y)$ $=(P U(t) x, y)=(u(t) x, y) \rightarrow 0$ as $t \rightarrow \infty$, so that $x \in W(D)$. This shows that $W(A) \cap H=W(D)$ and using the same fact for $A^{*}$ and $D^{*}$ and Part I, $W(D)=W\left(D^{*}\right)$ follows.

Part III. The completion of the lemma now follows by noting that if $L=S D S^{-1}, D$ dissipative, then $W(L)=S(W(D))$ and $\left[W\left(L^{*}\right)\right]^{\perp}$ $=S\left(\left[W\left(D^{*}\right)\right]^{\perp}\right)$. Since, by Part II, $W(D)=W\left(D^{*}\right)$ so that $W(D)$ $\cap\left[W\left(D^{*}\right)\right]^{\perp}=\{0\}$, we obtain $W(L) \cap\left[W\left(L^{*}\right)\right]^{\perp}=\{0\}$.

3. Construction of the counterexample. Let $H$ be the Hilbert space $L_{2}([0, \infty))$ and let $V(t)$ be right translation by $t$ on $H$, so for $t \geqq 0$,

$$
\begin{aligned}
V(t) f(x) & =f(x-t) \quad \text { when } x \geqq t, \\
& =0 \quad \text { otherwise, }
\end{aligned}
$$

and

$$
V^{*}(t) f(x)=f(x+t)
$$

It is easy to check that $V(t)$ and $V^{*}(t)$ are $\left(C_{0}\right)$ semigroups of operators whose norms are identically one.

For each $t>0$, define the integer $k_{0}=k_{0}(t)$ by requiring $4^{k_{0}}<t \leqq 4^{k_{0}+1}$. For each integer $k>k_{0}(t)$ define the interval $I_{k}(t)=\left(4^{k}-t, 4^{k}\right]$ and let $I_{k_{0}}(t)=\left[0,2 \cdot 4^{k_{0}}-t\right]$. Define the operator $P(t)$ on $H$ for each $t \geqq 0$ by $P(0)=0$ and

$$
\begin{aligned}
P(t) f(x) & =f\left(2 \cdot 4^{k}-t-x\right) \quad \text { when } x \in I_{k}(t), \quad k \geqq k_{0}(t), \\
& =0 \quad \text { otherwise. }
\end{aligned}
$$


By inspection $P(t)$ is well defined and $\|P(t)\| \leqq 1$ for all $t \geqq 0$.

Let $H_{2}=H \oplus H$ with the usual direct sum inner product: $\langle(f, g),(h, k)\rangle$ $=\langle f, h\rangle+\langle g, k\rangle$. Define the operators $S(t), t \geqq 0$, on $H_{2}$ by

$$
S(t)=\left(\begin{array}{cc}
V^{*}(t) & P(t) \\
0 & V(t)
\end{array}\right),
$$

i.e., $S(t)(f, g)=\left(V^{*}(t) f+P(t) g, V(t) g\right)$.

Since $\|V(t)\|=\left\|V^{*}(t)\right\|=1 \geqq\|P(t)\|$ for all $t \geqq 0$ it follows that $\|S(t)\| \leqq 2$ for all $t \geqq 0$. We now establish that $S(0)=I$ and that $S(t)$ has the semigroup property $S(u+t)=S(u) S(t)$ for all $u, t>0$. Since $P(0)=0$, we have $S(0)=I$. The semigroup property translates into the following equality:

$$
V^{*}(u) P(t) g(x)+P(u) V(t) g(x)=P(u+t) g(x)
$$

for all $g$ in $H$ and $u, t \geqq 0$, where the fact that $V(t)$ and $V^{*}(t)$ are semigroups has been used. Rewriting (I) with the definitions of $V(t)$, $V^{*}(t)$, and $P(t)$, we must prove that $h_{1}(x)+h_{2}(x)=h_{3}(x)$ where

$$
\begin{array}{rlrl}
h_{1}(x) & =g\left(2 \cdot 4^{k}-t-x-u\right) & \text { when } x+u \in I_{k}(t), \quad k \geqq k_{0}(t), \\
& =0 \quad \text { otherwise; } & & \\
h_{2}(x) & =g\left(2 \cdot 4^{k}-t-x-u\right) & \text { when } x \in I_{k}(u), \quad x \leqq 2 \cdot 4^{k}-t-u, \\
& =0 \quad \text { otherwise; } & & \\
& 0 k_{0}(u), \\
h_{3}(x) & =g\left(2 \cdot 4^{k}-t-x-u\right) & \text { when } x \in I_{k}(u+t), \quad k \geqq k_{0}(u+t), \\
& =0 \quad \text { otherwise. }
\end{array}
$$

Letting $g, u, t$, and $x$ be fixed but arbitrary, and noting that the arguments of $h_{1}, h_{2}$, and $h_{3}$ are equal, we need only verify that the conditions imposed on $x$ for these functions enable us to obtain the desired equality. Since the computations are not entirely obvious we include some of them, splitting the proof in to three parts.

(1) If $h_{1}(x) \neq 0$, then $h_{1}(x)=h_{3}(x)$. Indeed, if $h_{1}(x) \neq 0$, then $x+u \in I_{k}(t), k \geqq k_{0}(t)$. First suppose $k>k_{0}(t)$ so that $t \leqq 4^{k}$ and $x \in\left(4^{k}-t-u, 4^{k}-u\right]$ (and $\left.x \geqq 0\right)$. If $4^{k}-t-u \geqq 0$, then $u+t \leqq 4^{k}$ and $k>k_{0}(u+t)$ so that $x \in I_{k}(u+t)$ and $h_{3}(x)=h_{1}(x)$. If $4^{k}-t-u<0$, then $4^{k}<u+t \leqq 4^{k+1}$ (since $u \leqq 4^{k}$ and $t \leqq 4^{k}$ ) and $k_{0}(u+t)=k$. In this case, $4^{k}-t \geqq 0$ implies $2 \cdot 4^{k}-t-u \geqq 4^{k}-u$ so that $x \in I_{k_{0}}(u+t)$ and again $h_{1}(x)=h_{3}(x)$. Finally, suppose $k=k_{0}(t)$. Then $x \in\left[0,4^{k}-u-t\right]$ implies $k=k_{0}(u+t)$ and $x \in I_{k_{0}}(u+t)$, so $h_{1}(x)=h_{3}(x)$. Similar arguments show that if $h_{2}(x) \neq 0$, then $h_{2}(x)=h_{3}(x)$.

(2) Either $h_{1}(x)$ or $h_{2}(x)$ must be zero (for each fixed $x, t$, and $u$ ). For if both were nonzero we would have $x+u \in I_{k}(t)$ for some $k \geqq k_{0}(t)$ 
and $x \in I_{j}(u)$ for some $j \geqq k_{0}(u)$. If $k>k_{0}(t)$ and $j>k_{0}(u)$ then $\left(4^{k}-u-t, 4^{k}-u\right]$ and $\left(4^{j}-u, 4^{j}\right]$ clearly have empty intersection, yielding a contradiction. Likewise, if $k>k_{0}(t)$ and $j=j_{0}(u)$, we have $4^{j}<u \leqq 4^{j+1}$ and $u+t \leqq 2 \cdot 4^{i}$. Hence $t<4^{i}$, from which it follows that $k<j$ and $2 \cdot 4^{j}>4^{k}$ and thus $2 \cdot 4^{j}-u-t>4^{k}-u-t$. Once again $x+u \in I_{k}(t)$ and $x \in I_{j}(u)$ are incompatible. The remaining two cases in which $k=k_{0}(t), j>k_{0}(u)$ and $k=k_{0}(t), j=k_{0}(u)$ follow similarly and we conclude that either $h_{1}(x)$ or $h_{2}(x)$ must be zero.

(3) If $h_{3}(x) \neq 0$ then either $h_{1}(x)$ or $h_{2}(x)$ is nonzero. Here we have $x \in I_{k}(u+t), k \geqq k_{0}(u+t)$. If $k>k_{0}(u+t)$, then $k>k_{0}(u)$ and $k>k_{0}(t)$ so that $I_{k}(u+t)=I_{k}(u) \cup\left[x: x+u \in I_{k}(t)\right]$. Thus one of $h_{1}(x)$ and $h_{2}(x)$ must be equal to $h_{3}(x)$ and hence nonzero. Finally if $k=k_{0}(u+t)$, then $x \in I_{k_{0}}(u+t)$. If either $k_{0}(t)=k_{0}(u+t)$ or $k_{0}(u)=k_{0}(u+t)$ then $x \in I_{k_{0}}(t)$ or $x \in I_{k_{0}}(u)$ and $h_{1}(x)$ or $h_{2}(x)$ is nonzero. If both $k_{0}(t)$ $<k_{0}(u+t)$ and $k_{0}(u)<k_{0}(u+t)$, then $I_{k}(u+t) \subseteq I_{k}(u) \rightarrow\left[x: x+u \in I_{k}(t)\right]$ and again one of the $h_{1}(x)$ and $h_{2}(x)$ must be nonzero.

Taking (1), (2), and (3) together we obtain $h_{1}(x)+h_{2}(x)=h_{3}(x)$ so that $S(t)$ is a semigroup.

To show that $S(t)$ is a $\left(C_{0}\right)$ semigroup we must show that for each $(f, g)$ in $H_{2}, S(t)(f, g)$ is continuous in $t$ on $[0, \infty)$. By the semigroup property this reduces to showing that $\|S(t)(f, g)-(f, g)\| \rightarrow 0$ as $t \rightarrow 0$. To this end we have

$$
\begin{aligned}
\|S(t)(f, g)-(f, g)\|^{2} & =\left\|\left(V^{*}(t) f+P(t) g-f, V(t) g-g\right)\right\|^{2} \\
& =\left\|V^{*}(t) f+P(t) g-f\right\|^{2}+\|V(t) g-g\|^{2} \\
& \leqq 2\left\|V^{*}(t) f-f\right\|^{2}+2\|P(t) g\|^{2}+\|V(t) g-g\|^{2} .
\end{aligned}
$$

The first and third terms of the last line get small as $t \rightarrow 0$ by the continuity of translation on $L_{2}([0, \infty)$ ) (or equivalently since $V(t)$ and $V^{*}(t)$ are $\left(C_{0}\right)$ semigroups). Also $\|P(t) g\| \rightarrow 0$ because $\|P(t) g\|=\|X(t) g\|$ where $X(t)$ is multiplication by the characteristic function of $\bigcup_{k=k_{0}(t)}^{\infty} I_{k}(t)$, and $\|X(t) g\| \rightarrow 0$ by the Lebesgue dominated convergence theorem.

Thus $S(t)$ is a uniformly bounded $(\|S(t)\| \leqq 2$ for all $t \geqq 0)\left(C_{0}\right)$ semigroup and by the general theory of $\left(C_{0}\right)$ semigroups, $S(t)$ has an infinitesimal generator $L$ defined for $y \in H_{2}$ by

$$
L y=\lim _{t \downarrow 0} \frac{S(t) y-y}{t} \quad \text { whenever this limit exists. }
$$

ThEOREM. There exists a uniformly bounded $\left(\mathrm{C}_{0}\right)$ semigroup $S(t)$ whose generator $L$ is not similar to a dissipative operator.

Proof. In view of Lemma 1 it suffices to show that 


$$
W(L) \cap\left[W\left(L^{*}\right)\right]^{\perp}=\{0\} .
$$

Setting $g=$ characteristic function of $(0,1)$ and defining $R$ $=\left\{2 \cdot 4^{k}-1: k=0,1,2, \cdots\right\}$, the definition of $P(t)$ shows that $P(r) g(x)=g(x)$ for all $r \in R$. Indeed $I_{k_{0}}(r)=[0,1]$ for such $r$. Now using the fact that $\langle V(r) g, h\rangle \rightarrow 0$ as $r \rightarrow \infty$ for all $h \in H$,

weak limit $(r \rightarrow \infty, r \in R) S(r)(0, g)$

$$
=\text { weak limit }(r \rightarrow \infty, r \in R)(g, V(r) g)=(g, 0) .
$$

Also for $(h, k) \in W\left(L^{*}\right)$,

$$
\begin{aligned}
\langle(g, 0),(h, k)\rangle & =\operatorname{limit}(r \rightarrow \infty, r \in R)\langle S(r)(0, g),(h, k)\rangle \\
& =\operatorname{limit}(r \rightarrow \infty, r \in R)\left\langle(0, g), S^{*}(r)(h, k)\right\rangle \\
& =0
\end{aligned}
$$

by the definition of $(h, k) \in W\left(L^{*}\right)$. Thus $(g, 0) \in\left[W\left(L^{*}\right)\right]^{\perp}$ and clearly $(g, 0) \in W(L)$, so $(g, 0) \in W(L) \cap\left[W\left(L^{*}\right)\right]^{\perp}$ and $L$ is not similar to a dissipative operator.

An investigation into the nature of the generator $L$ of $S(t)$ yields the following interesting result. The domain of $L$ consists of all $(f, g) \in H_{2}$ for which

(1) $g$ is (modulo null functions) absolutely continuous on $H$, $\lim _{x \downarrow_{0}} g(x)=0$, and $d g / d x \in H$.

(2) $f$ is absolutely continuous everywhere except possibly at the points $4^{k}, k$ integral.

(3) Letting $h(x)=f(x)+\sum_{4 k<x} g\left(4^{k}\right)$, then $d h / d x \in H$, so that the jumps of $f$ at $4^{k}$ are the negatives of the values $g\left(4^{k}\right)$.

For $(f, g) \in$ Domain $(L), L(f, g)=(d h / d x,-d g / d x)$.

In conclusion, the counterexample presented is a $\left(C_{0}\right)$ semigroup with unbounded generator. This leaves open the question of whether or not the (bounded) generator of a uniformly bounded, uniformly continuous semigroup must be similar to a dissipative operator.

\section{REFERENCES}

1. S. R. Foguel, A counterexample to a problem of Sz.-Nagy, Proc. Amer. Math. Soc. 15 (1964), 788-790.

2. P. R. Halmos, On Foguel's answer to Nagy's question, Proc. Amer. Math. Soc. 15 (1964), 791-793.

3. R. S. Phillips, Dissipative operators and hyperbolic systems of partial differential equations, Trans. Amer. Math. Soc. 90 (1959), 193-254.

4. F. Riesz and B. Sz.-Nagy, On extensions of linear transformations on a Hilbert space which extend beyond that space, Appendix to Functional Analysis, Ungar, New York, 1960.

Reed College 